\title{
Justification of the active method of protecting the insulation of submersible electric motors
}

\author{
V. A. Trushkin ${ }^{1, *}, O . N$. Churlyaeva $^{1}$, and $R . V$. Kozichev $^{1}$ \\ ${ }^{1}$ Saratov State Agricultural University n. a. N.I. Vavilov, Saratov, Russia
}

\begin{abstract}
The article provides an analysis of the properties of the working environment of submersible electrical equipment. The influence of the operating parameters of electrical equipment on the physical properties of the fluid (its electrically conductive properties) is considered. Mathematical confirmation of the implementation of electroosmosis in the capillaries of the insulation of submersible electric motors is given. The rationale for the active method of protecting submersible electrical equipment from reducing insulation resistance and preventing electrical breakdown is given.
\end{abstract}

\section{Introduction}

Polymeric materials are widely used in industry in the form of coatings and whole parts. They are solid insulating materials. Many varieties of polymers have been developed, but all of them are characterized by an undesirable aging process, worsening their durability, appearance and strength. With aging, the structure and chemical composition of polymeric materials change.

In electric motors of submersible electric pump installations, as well as in electric motors of general industrial design, the main operational parameter is insulation resistance [1].

Considering the operational features, the main type of insulation for submersible electric motors are polymers, which are represented by polyethylene (LDPE and HDPE) and polyvinyl chloride (PVC). These materials are characterized by high electrical strength; have extremely low hygroscopicity; possess high chemical resistance to alkalis, acids and solvents; have high elasticity and low thermal conductivity. Winding wires of submersible electric motors are represented by PVDP, PEVVP, PEP-V, PEPT-V, etc. brands, the insulation of which is two-layer [2, 3].

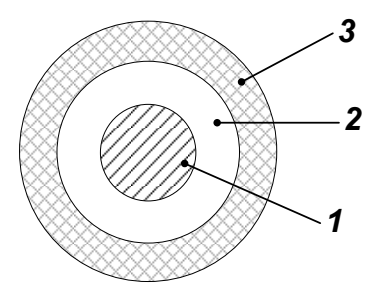

Fig. 1. Insulation winding wire for submersible electric motors: 1-conductor; 2- insulation (LDPE, varnish PE-939); 3- shell (HDPE, PVP).

\section{Characteristics of the working environment}

The environment for submersible electropump equipment is water, the electrical conductivity of which depends on the concentration of dissolved mineral salts and temperature [4]. Natural waters are mainly solutions of mixtures of strong electrolytes. The mineral part of the water is composed of $\mathrm{Na}+, \mathrm{K}+, \mathrm{Ca} 2+, \mathrm{Cl}-, \mathrm{SO} 42-$, $\mathrm{HCO} 3-$ ions, the presence of which determines the electrical conductivity of natural waters $[4,5,6]$. The presence of other ions in water, for example, $\mathrm{Fe} 3+, \mathrm{Fe} 2$ ,$+ \mathrm{Mn} 2+, \mathrm{Al} 3+$, NO3-, HPO4-, H2PO4- affects the electrical conductivity if they are found in significant quantities. On this basis, the specific conductivity of water (electrolyte solution) will be determined by the number of ions that carry electricity and the speed of their migration $[3,4]$.

The dependence of the electrical conductivity of dilute solutions on temperature is described by an empirical equation $[4,7]$ :

$$
\chi=\alpha_{\mathrm{i}} \mathrm{CF}\left(\mathrm{U}_{+}+\mathrm{U}_{-}\right)[\mathrm{S}]
$$

where, $\alpha_{i}$ - the degree of dissociation of the electrolyte; C - molar concentration of equivalent, $\mathrm{mol} / \mathrm{m}^{3}$; $\mathrm{F}$ is the Faraday number, $96485 \mathrm{C} / \mathrm{mol} ; \mathrm{U}_{+}, \mathrm{U}_{\text {- }}$ absolute speeds of movement of the cation and anion (velocity with a field potential gradient equal to $1 \mathrm{~V} / \mathrm{m}$ ), $\mathrm{m}^{2} \mathrm{~V}^{-1} \mathrm{~s}^{-1}$.

\section{Description of the mechanism of insulation destruction of submersible electric motors}

During equipment operation, polymer insulation of submersible electric motors is subjected to thermal,

\footnotetext{
*Corresponding author: v.a.trushkin@mail.ru
} 


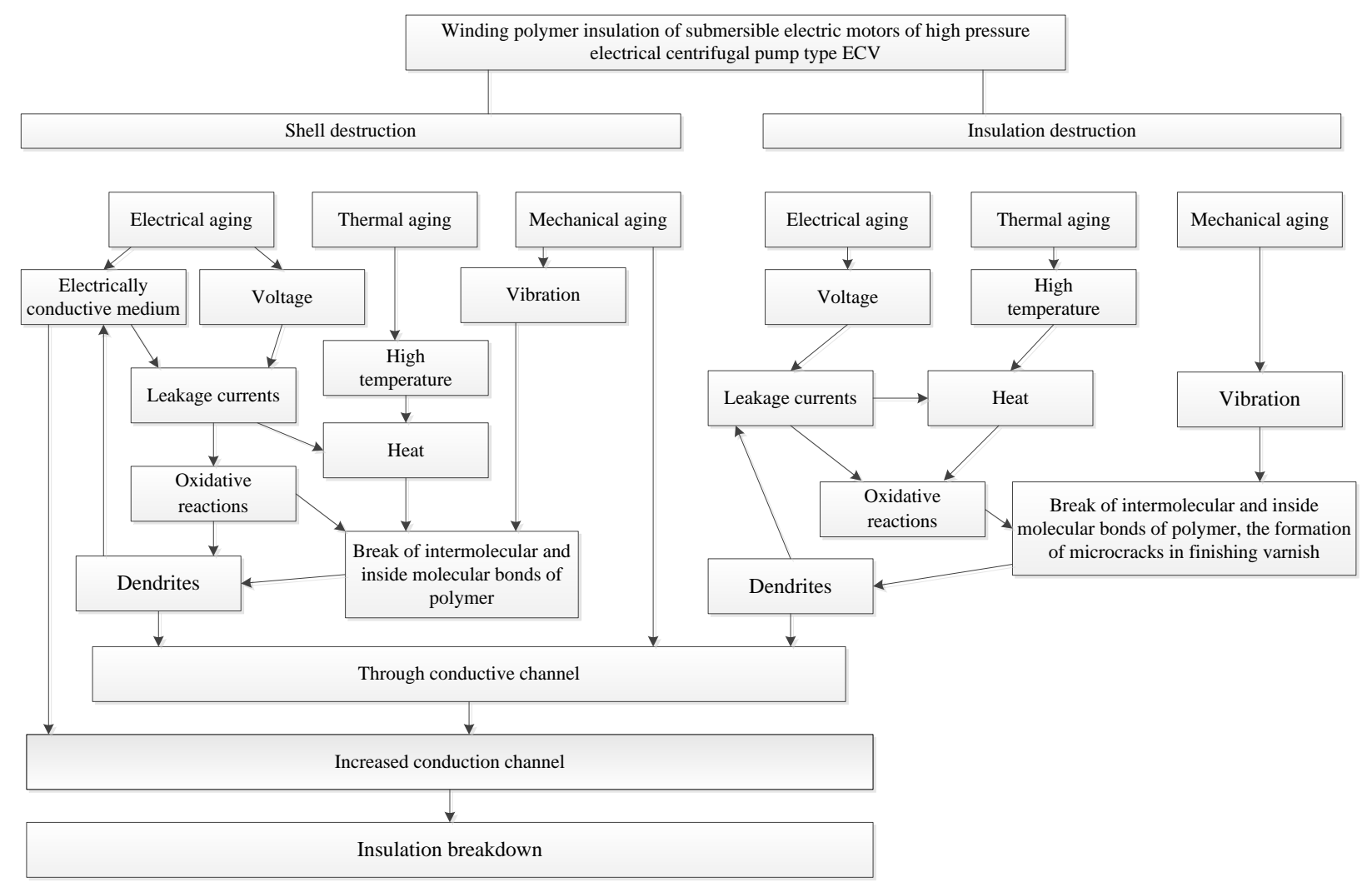

Fig. 2. The scheme of destruction of polymer insulation of winding wires of submersible electric motors.

electrical, mechanical, and to a lesser extent other types of effects. A feature of this equipment is that all negative factors simultaneously have a damaging effect $[8,9,10]$.

The most negative impact on the state of the insulation has thermal destruction. The temperature rise occurs mainly due to improper operation, not performing the routine work specified in the regulatory documentation. Also, the water in the submersible pumping equipment plays the role of a heat sink. In [1] the minimum permissible velocity of the fluid through the intake ports is determined and is $0.5 \ldots 1.0 \mathrm{~m} / \mathrm{s}$. With a decrease in the velocity of the fluid, the temperature of the windings of the submersible electric motor will begin to rise and may exceed the maximum allowable threshold for this type of equipment [1].

An increase in temperature leads to accelerated destruction of the insulation and a change in the electrically conductive properties. The environmental resistance depending on temperature can be written by the expression $[3,4,10]$ :

$$
\rho=\rho_{o}(1+\alpha t)[\Omega]
$$

The result of heat exposure is a break in the intermolecular, and to a lesser extent within the molecular bonds. The combination of certain types of effects, primarily thermal and to a lesser extent mechanical, leads to the formation of voids, and further to the formation of branched channels with a complex and branched structure. Taking into account the environmental properties for the equipment in question, the further destruction of the integrity of the insulation leads to the formation of a through channel filled with liquid $[8,11,12,13]$.

These microchannels can be considered as homogeneous parts of an electrical circuit with certain parameters (electrical and specific resistance, crosssection, length). With the further development of damage, the dimensions of the channels change, and when a bandwidth of one of the electrically conductive channels reaches certain parameters, the current through it increases incrementally. Electrical breakdown of insulation to the motor housing takes place [14]. Figure 2 shows a diagram of the formation of through conductive channels, taking into account the main destructive factors affecting the integrity of the insulation.

\section{Justification of the method of increasing operational insulation}

To increase the operational reliability of polymer insulation, passive, active and combined methods of protection are used. Passive methods of protecting polymers are used at the stage of their production and apply stabilizer additives, acceptors of free radicals, acceptors of active aging products, light stabilizers, antioxidants, antiozonants, flame retardants, anticontaminants with mechanical action, corrosion inhibitors and biocides with stabilizing properties. Active protection methods reduce the effects of aging factors. As a way to increase the resistance of polymer insulation from electrical breakdown in an electrically conductive medium, one can propose a method based on 
the rupture of electrically conductive channels using electroosmosis [2, 3].

This phenomenon was used by Nemirovsky A.E. to increase the insulation resistance of the windings of electric air-cooling machines in conjunction with other additional methods (blowing warm air on the windings, heating from an external heat source, etc.) [15].

The essence of the proposed method is to create a potential difference, using a constant current source, between the winding of the submersible electric motor and the body of the electric machine during technological breaks.

Modern ideas about the mechanism of electroosmosis are based on the idea of the existence of a double electrostatic layer, at the interface of the liquid and solid phases. A double electrostatic layer appears on the border between the inner surface of the microchannel and the adjacent surface of the water. In this case, the surface layer of water is positively charged, and the inner surface of the hollow rod is negative. The sign of the charge that the surface layer receives is determined by the ratio of the values of the dielectric constant of materials $[8,14]$.

Electrostatic double layer is fixed bed. A less dense layer adjoins the surface layer of positive ions, which is able to move under the action of an electric field to the negative electrode. For this, the positive pole of the power source is fed to the winding of the submersible electric motor, and negative is fed to the body of the submersible electric motor. As it moves, this layer in the form of a cylindrical shell entrains ion-molecular bulk complexes (clusters) and free water molecules. Since the working environment for the equipment under consideration has electrical conductivity, it should be noted that in electrolytes the mechanism of movement of charge carriers (ions) is thermally activated, that is, they can move by themselves due to an increase in temperature, and the application of an external electric field will set the drift direction $[7,13,16,18]$.

The speed of an electroosmotic phenomenon is written by the formula $[7,11,17]$ :

$$
u=\frac{z z_{0} \sqrt{\xi}}{\eta z_{\gamma}}
$$

where, $\mathrm{u}$ - the volumetric rate, $\mathrm{m}^{3} / \mathrm{s}$; I - current strength, A; $\eta$ - the coefficient of viscosity; $\chi$ - conductivity, $1 / \Omega \cdot \mathrm{m} ; \xi$ - electrokinetic zetapotential.

Representing a single capillary channel in the form of a homogeneous section of the circuit, according to Ohm's law, the current flowing through this section is determined by the expression:

$$
I=\frac{U}{R}
$$

where, I - current in the circuit, A; U - voltage on the chain section, $\mathrm{V} ; \mathrm{R}$ - the resistance of the considered section of the circuit, $\Omega$.

The resistance of the circuit is expressed through its resistivity:

$$
R=\rho \frac{l}{S}
$$

where, $\rho$ - the resistivity of the circuit, $\Omega / \mathrm{m}$; 1 - the length of the considered section, $\mathrm{m} ; \mathrm{S}$ - effective conductor cross-section, $\mathrm{mm}^{2}$.

Taking into account the expression (2) and (5), we obtain the resistance value of the chain section taking into account the effect of temperature on the electrical properties of the conductor material, in this case the electrolyte

$$
R=\rho_{0}(I+a t) \frac{l}{S}
$$

The resulting expression (6) is substituted in (5)

$$
I=\frac{L S}{\rho_{0} /(+a t j) i d}
$$

Substituting the expressions (1) and (7) into (3), we obtain the formula for the speed of an electroosmotic phenomenon in electrolytes, taking into account the operating conditions of the submersible electric pump equipment:

$$
u=\frac{\varepsilon \varepsilon_{0} \frac{U S}{\rho_{0}[1+\alpha t) l^{5}}}{\eta \alpha_{i} C F(U++U-)}
$$

Simplified expression (8):

$$
u=\frac{\varepsilon \varepsilon_{0} U S \xi}{\left(\eta \alpha_{i} C F(U++U-)\right)\left(\rho_{0}(1+\alpha t) l\right)}
$$

The obtained expression (9) shows the dependence of the flow rate of the electroosmotic process taking into account the electrically conductive properties of the working medium, the parameters of the electrically conductive channel and the magnitude of the applied voltage. Analyzing this expression, one can see that a change in the magnitude of the applied voltage allows changing the speed of the flow of electroosmotic processes in the microchannels of polymer insulation. It should be borne in mind that the total leakage current will be the sum of leakage currents through the insulation and leakage currents through the formed microchannels filled with water. In this case, the probability of formation of a stable electrically conductive channel is possible in the place of the insulating system of the submersible electric motor, where the resistance of the working medium is minimal, namely in the place of the output of the winding frontal part from the stator slots.

\section{Conclusion}

Submersible electric pump installations are characterized by simplicity of design and high structural reliability. However, operational reliability remains very low. The average service life is no more than 3-4 years and depends on external factors and operating conditions. Therefore, the most urgent direction at the present time is the solution of questions related to the development of methods and technical solutions, which make it possible to significantly improve the characteristics of equipment already in operation. 
The development of the method of active protection may be one of such methods and will extend the service life of the submersible electric pump equipment, which can positively affect the technical condition of the submersible pump unit as a whole and will partially solve the issue of improving the operational reliability of this equipment.

\section{References}

[1] Centrifugal electric pump unit. Well submersible ETSV. Manual. H49.872.00.00.000 OM

[2] V.A. Trushkin, R.V. Kozichev, 2019 Diagnosing the insulation of submersible water supply equipment with the implementation of electroosmotic moisture protection Technique and equipment for the village 1, 30-32

[3] M.M. Mikhailov, 1960 The moisture permeability of organic dielectrics ed. W.T. Renne. (Moscow; Leningrad: Gosenergoizdat) 163

[4] A.D. Simon 2003 Physical chemistry: a textbook for high schools (Moscow: Agar) 320

[5] GOST R 51232-98 2005 Drinking water. General methods for the organization and methods of quality control (Moscow: Gosstandart of Russia) 18

[6] G.N. Zatsepina (1973) Properties and structure of water (Moscow: Moscow University Press) 163

[7] T.A. Jankowski, F.C. Prenger, D.D. Hill, S.R. O'Bryan, K.K Sheth, E.B. Brookbank, D.F. Hunt, Y.A. Orrego Development and validation of a model for electric induction motors IEEE Transactions on Industrial Electronics 57(12), 4043-4054 (2010)

[8] Ushakov V.Ya. 1988 Electrical aging and resource monolithic polymer insulation (Moscow: Energoatomizdat) 152

[9] Yu. Kang, Ch. Yang, X. Huang, AC electroosmosis in microchannels packed with a porous medium Journal of Micromechanics and Microengineering 14(8), 1249-1257 (2004)

[10] Miller R.D. Neglected aspects of electroosmosis in porous bodies Science 122(3165), 373-374 (1955)

[11] N.A. Mishchuk, T. Heldal, T. Volden, J. Auerswald, H. Knapp, Microfluidic pumping of the second kind Microfluidics and Nanofluidics 11(6), 675684 (2011)

[12] J.D. Sherwood, M. Mao, S. Ghosal, Electroosmosis in a finite liter of the colloids 30(31), 9261-9272 (2014)

[13] I.S. Sukhachev, V.V. Sushkov, Submersible electric motor system at the West Siberia oil fields IEEE Conference Dynamics of Systems, Mechanisms and Machines DOI:10.1109/Dynamics.2016.7819090 (2016)

[14] V.Ya. Antonchenko, A.S. Davydov, V.V. Il'in, Fundamentals of water physics (Kiev: Scientific thought) 672 (1991)

[15] A.E. Nemirovsky, Improving the efficiency of drying and moisture protection of insulation of electric motors used in agriculture, based on the intensification of electroosmotic phenomena: author. diss. doc tech. sciences (Saint-Petersburg, Pushkin) 50 (1993)
[16] W. Liguo, W. Songlin, A. Tianqi, X. Dianguo, Study on suppressing temperature. International conference on technological advances in electrical, Electronics and Computer engineering, Taeece (2013)

[17] C.J. Tsai, K.D. Jordan Fused Cubic Structures for $(\mathrm{H} 2 \mathrm{O}) \mathrm{n}, \mathrm{n}=8,12,16$ and 20 Journal of Physical Chemistry 97, 5208-10 (1993)

[18] F.A. Belousov, The influence of the electric field on the diffusion of water in polymer insulation: dis. cand. tech. sciences (Saint-Petersburg) 183 (2000) 